\title{
O Estado da Arte das Pesquisas sobre o Pensamento Algébrico com Crianças ${ }^{1}$
}

\section{The State-Of-The-Art of Research on Algebraic Thinking with Children}

\author{
Vinicius Carvalho Beck \\ viniciusbeck@,furg.br \\ João Alberto Silva \\ joaosilva@,furg.br
}

\begin{abstract}
Resumo
O objetivo deste trabalho é apresentar os principais resultados das pesquisas recentes sobre o pensamento algébrico com crianças. Pode-se dizer que embora tenham surgido muitos trabalhos abordando a temática do pensamento algébrico nos últimos anos, prevalece a ramificação estabelecida por Blanton e Kaput (2008), que divide o pensamento algébrico em duas vertentes: a Aritmética Generalizada e o pensamento funcional. A primeira se caracteriza pela generalização das operações e o raciocínio acerca da relação entre números. Já a segunda se caracteriza pela descrição da variação numérica em certo domínio. Além desta divisão em vertentes, muitos trabalhos também apresentam a ideia de que problemas que envolvem sequências e combinações potencializam a habilidade de pensar algebricamente.
\end{abstract}

Palavras-chave: Pensamento Algébrico. Aritmética Generalizada. Pensamento Funcional.

\begin{abstract}
The aim of this paper is to present the main results of the recent studies on algebraic thinking with children. We could say that although they have arisen many studies addressing the subject of algebraic thinking in recent years prevails the branch established by Blanton and Kaput (2008), which divides the algebraic thinking in two ways: the Generalized Arithmetic and functional thinking. The first is characterized by the generalization of the operations and the reasoning about the relationship between numbers. The second is characterized by the description of the numerical variation in right field. In addition to this division into strands, many papers also feature the idea that problems involving sequences and combinations to maximise the ability to think algebraically.
\end{abstract}

Keywords: Algebraic Thinking. Aritmética Generalizada. Functional Thinking.

\section{Introdução}

Explicar como o pensamento lógico-matemático se desenvolve tem sido um grande desafio para os estudiosos das Ciências Cognitivas, sobretudo a partir da segunda metade do século XX. Jean Piaget $(1937,1950,1977)$ foi um dos primeiros pesquisadores a estudar de forma mais detalhada o processo de aprendizagem da Matemática na infância. Em seu trabalho, ele 
apresentou estudos experimentais no âmbito da Psicologia, a partir dos quais pôde construir um modelo da organização do pensamento lógico-matemático da criança. Alguns de seus alunos de doutorado, como Constance Kamii (1990) e Gérard Vergnaud (1985, 1990, 1997), deram prosseguimento às suas pesquisas neste sentido.

Nos últimos anos, surgiram na literatura trabalhos abordando o desenvolvimento do pensamento algébrico (BLANTON e KAPUT, 2005; CARPENTER, LEVI, FRANKE e ZERINGUE, 2005; IRWIN e BRITT, 2006; CANAVARRO, 2007; FUJII e STEPHENS, 2008; STEPHENS e WANG, 2008).

Alguns documentos que embasam a prática docente já apresentam uma expectativa de se desenvolver o pensamento algébrico desde anos iniciais do Ensino Fundamental (NCTM, 2000; BRASIL, 2012). O objetivo deste trabalho é apresentar os principais resultados das pesquisas sobre o pensamento algébrico com crianças.

Na seção 2 apresentamos nosso referencial de concepção sobre um estado da arte. Na seção 3 são apontadas algumas caracterizações de pensamento algébrico. Na seção 4 são apresentados os principais resultados daquilo que muitos pesquisadores têm chamado de Aritmética Generalizada. Na seção 5 são relatados os principais resultados relacionados com o pensamento funcional. Na seção 6 são feitas considerações finais.

\section{O que é um estado da arte?}

Vários estudos científicos se destinam a temáticas panorâmicas, ressaltando aspectos mais gerais constatados pela leitura de textos que abordam uma temática em comum. Trata-se de levantamentos bibliográficos, comumente chamados de estados da arte. Consideremos a definição proposta por Romanowski e Ens (2006):

\footnotetext{
Um estado da arte pode constituir-se em levantamentos do que se conhece sobre determinada área, desenvolvimento de protótipos de análises de pesquisas, avaliação da situação da produção do conhecimento da área focalizada [...]. Pode, também, estabelecer relação entre produções anteriores, identificando temáticas recorrentes e apontando novas perspectivas, consolidando uma área de conhecimento e constituindo-se orientações de práticas pedagógicas para a definição de parâmetros de formação de profíssionais [...]. Pode, ainda, verificar na multiplicidade e pluralidade de enfoques e perspectivas, indicativos para esclarecer e resolver as problemáticas históricas [...]. Igualmente torna possível reconhecer a importância da investigação, os aportes significativos da construção da teoria e prática pedagógica [...]. (ROMANOWSKI e ENS, 2006, p.41).
}

No caso do presente trabalho, o estado da arte é constituído pelo levantamento do que se conhece sobre o tema da pesquisa e pela relação entre produções anteriores para indicar novas perspectivas para as pesquisas sobre o pensamento algébrico com crianças. Considerou-se 
principalmente os trabalhos produzidos a partir de 2003, embora também sejam citados alguns referenciais anteriores a este período, bem como documentos oficiais que nortearam boa parte dessas pesquisas.

Inicialmente, foram feitas consultas nas bases de dados da Coordenação de Aperfeiçoamento de Pessoal Superior (CAPES) e no Scientific Eletronic Library On Line (SciELO). No entanto, constatamos que alguns trabalhos muito importantes sobre o pensamento algébrico não foram encontrados (BLANTON e KAPUT, 2005; FUJII e STEPHENS, 2008; KIERAN, 2007). Assim, optou-se por realizar buscas diretamente nos periódicos da área da Educação, especialmente aqueles nos quais predominam produções no campo da Educação Matemática. Em uma primeira procura, realizada diretamente em sites de busca da internet, o trabalho de Canavarro (2007) foi encontrado. Ele contém várias citações de artigos bastante relevantes sobre o pensamento algébrico, e também situa o leitor sobre as principais pesquisas realizadas até então. Além disso, ele também indicou os periódicos que poderiam conter mais trabalhos versando sobre o mesmo tema.

Foram feitas buscas nos seguintes periódicos: Journal for Research in Mathematics Education (qualis A1), Educação Matemática Pesquisa (qualis B2), Estudos em Avaliação Educacional (qualis A2), Quadrante (qualis B1), ZDM - The International Journal on Mathematics Education (qualis A1), Boletim GEPEM (qualis B1), Education Studies in Mathematics (qualis A2), Revista Latinoamericana de Investigación em Matemática Educativa (não informado), Revista Eletrônica de Educação (B1), International Journal for Research in Mathematics Education (qualis B2) e Recherches em Didactique des Mathématiques (não informado). A consulta sobre o qualis de cada periódico foi feita no sistema webqualis (CAPES, 2015), tomando como referência o parâmetro Educação no campo Área de Avaliação.

Além dos trabalhos consultados nos periódicos, também foram incluídas dissertações, teses e trabalhos publicados em congressos que de alguma forma se relacionam com o tema. Não houve uma busca específica no sentido de encontrar estes últimos trabalhos. $\mathrm{O}$ critério foi simplesmente afinidade com o tema da pesquisa. Eles foram escolhidos na medida em que eram considerados relevantes para compor este estado da arte, por abordarem questões relativas ao pensamento algébrico nas etapas iniciais da Educação Matemática.

\section{Pensamento Algébrico}

Segundo Ifrah (1998), uma das ideias matemáticas mais remotas é a ideia de contagem, que inicialmente foi utilizada para correspondência de objetos concretos. Mais tarde, as exigências do meio fizeram com que fossem utilizadas novas representações. $O$ avanço nestas 
representações evoluiu em termos de linguagem, até o aparecimento dos primeiros sistemas simbólicos algébricos.

A palavra Álgebra se originou da palavra árabe $a l-j a b r$, a qual foi utilizada no livro Al-Kitab al-jabr wa'l Muqabalah, escrito por Mohammed ibn-Musa Al-Khwarizmi por volta 825D.C., que apresentava alguns métodos de resolução de equações, e por isto, o termo Álgebra passou a ser referente ao estudo das equações (BAUMGART, 1992).

Nos últimos anos, muito tem se discutido no cenário das pesquisas em Educação Matemática a respeito do conceito de pensamento algébrico (BLANTON e KAPUT, 2005; CARPENTER et al., 2005; SOARES, BLANTON e KAPUT, 2006; IRWIN e BRITT, 2006; CANAVARRO, 2007; KAPUT, CARRAHER e BLANTON, 2007; FUJII e STEPHENS, 2008; STEPHENS e WANG, 2008; CARRAHER, MARTINEZ e SCHLIEMANN, 2008; GREENES e RUBENSTEIN, 2008; MOLINA, 2009). A seguir, apresenta-se algumas definições deste tipo de pensamento encontradas na literatura.

Blanton e Kaput (2005, p.413) caracterizam o pensamento algébrico como o "processo pelo qual os alunos generalizam ideias matemáticas a partir de um conjunto de casos particulares, estabelecem essas generalizações através de discurso argumentativo, e expressam-nas de formas progressivamente mais formais e adequadas à sua idade". Estes autores desenvolveram pesquisas com o objetivo de compreender e caracterizar o pensamento algébrico nos primeiros anos escolares, definindo este início da aprendizagem de conceitos algébricos como early algebra.

Considerar a generalização, a argumentação e a expressão como partes constituintes do processo de aprendizado da Álgebra é admitir que o aprendizado algébrico não está restrito exclusivamente à compreensão dos símbolos e manipulação de expressões envolvendo incógnitas e variáveis, mas também deve contemplar formas de pensar mais generalistas, argumentativas e com maior poder de representação de ideias matemáticas, o que amplia consideravelmente o horizonte de contextos nos quais o pensamento algébrico pode desempenhar algum papel importante.

Verschaffel, Greer e De Corte (2007) entendem que o pensamento algébrico está associado com o reconhecimento do que é geral numa situação matemática e à expressão de generalizações. Kieran (2007) ressalta que a Álgebra não deve ser entendida apenas como um conjunto de procedimentos envolvendo símbolos alfabéticos, que não deve ser encarada apenas como um conjunto de técnicas, mas também como uma forma de pensar e raciocinar em situações matemáticas. Apesar de Kieran (2007) utilizar o termo Álgebra, e não pensamento algébrico, a Álgebra de Kieran (2007) parece estar definida no mesmo sentido do pensamento algébrico de Verschaffel et al. (2007), e também de Blanton e Kaput (2005). 
Linz e Gimenez (1996) ressaltam que o conceito de pensamento algébrico no meio acadêmico não é compreendido da mesma forma por todos os profissionais que de alguma forma trabalham com a Álgebra, o que acaba por influenciar muitos professores a caracterizar a Álgebra como uma área na qual se trabalha exclusivamente a habilidade de calcular com letras, atribuindo mais importância à sintaxe do que ao pensamento da criança.

A ideia de se trabalhar com o pensamento algébrico nas etapas iniciais da Educação Escolar é relativamente recente. O National Council of Teachers of Mathematics (NCTM, 2000) destaca quatro eixos que devem orientar o trabalho pedagógico envolvendo o pensamento algébrico nos vários níveis de ensino. São eles: “(1) compreender padrões, relações e funções; (2) representar e analisar situações e estruturas matemáticas usando símbolos algébricos; (3) usar modelos matemáticos para representar e compreender relações quantitativas; e (4) analisar a mudança em vários contextos". Cada nível de ensino deve considerar também aspectos específicos da faixa etária dos alunos e dos conteúdos de outros eixos da Matemática, recebendo adequações de acordo com estas características (NCTM, 2000).

Logo em seguida da publicação das orientações do NCTM (2000), surgiram na literatura vários trabalhos abordando questões relativas ao desenvolvimento do pensamento algébrico, inclusive no Brasil.

Falcão (2003) questiona a anterioridade da Aritmética em relação à Álgebra, destacando que a Álgebra é um campo com características específicas, e não simplesmente uma extensão da Aritmética. O autor conclui que a Álgebra no Ensino Fundamental não deve se restringir apenas à aquisição de códigos algorítmicos ao final desta etapa de ensino, destacando que as noções de função e incógnita poderiam ser mais bem desenvolvidas se estes assuntos fossem abordados com maior frequência e desde o Ciclo de Alfabetização. Um termo utilizado pelo autor que merece destaque, tendo em vista a proposta de Álgebra na alfabetização, é o que ele chama de pré-Álgebra, ou seja, uma primeira experiência com o pensamento algébrico, sem a exigência rigorosa de uma representação simbólica.

No entanto, abordar uma pré-Álgebra trouxe para a comunidade de educadores matemáticos diversas novas questões, pois pouco se discutia sobre o pensamento algébrico na alfabetização. Aliás, por se tratar de um eixo estruturante recente, até mesmo a formulação de tarefas e situações-problema não são claramente diferenciadas de outros eixos na literatura.

Os primeiros problemas propostos abordando o pensamento algébrico ressaltavam a ideia de regularidade e previsibilidade, na maioria das vezes estando relacionados com sequências e combinações.

Gomes (2003) apresenta e recomenda diversas situações algébricas para o Ensino Fundamental envolvendo sequências e combinações, as quais podem servir como base para a 
criação e manipulação de equações e expressões algébricas. A autora afirma que a Álgebra pode ser abordada desde o início do Ensino Fundamental, destacando a ideia de uma alfabetização algébrica, e ainda evidencia as relações que podem ser traçadas entre a Álgebra, a Geometria e a Aritmética.

Fujii (2003) utiliza a expressão quase-variável para designar um número ou conjunto de números numa expressão que revelam uma relação matemática e que se manterá independentemente dos números a serem posteriormente utilizados. Isto é, o autor define uma quase-variável como uma sequência que apresenta regularidade e previsibilidade.

Como se pode notar, tendo em vista os trabalhos de Falcão (2003), Gomes (2003) e Fujii (2003), em um primeiro momento os trabalhos sobre o pensamento algébrico estavam mais ligados ao estabelecimento de uma constituição terminológica para os conceitos envolvidos, estando centrados nas ideias de regularidade e previsibilidade, com situações que envolviam basicamente sequências e combinações de objetos. Com base nas tarefas abordadas nestes primeiros estudos, pode-se definir situações envolvendo combinações como aquelas nas quais uma contagem de objetos é requerida, na qual a ordem destes objetos é alterada ou constitui uma informação relevante para a determinação da estratégia de resolução.

Blanton e Kaput (2005) afirmam que o pensamento algébrico se subdivide em duas vertentes: a Aritmética Generalizada e o pensamento funcional. A primeira se caracteriza pela generalização das operações e o raciocínio acerca da relação entre números. Já a segunda se caracteriza pela descrição da variação numérica em certo domínio.

No Brasil, apenas recentemente o pensamento algébrico passou a fazer parte das orientações curriculares em nível nacional. Em 2012 o Ministério da Educação (MEC) publicou um documento sobre os direitos de aprendizagem no Ciclo de Alfabetização (BRASIL, 2012). Segundo este documento, que é o primeiro a citar o pensamento algébrico como um dos eixos, o conhecimento matemático nos anos iniciais deve ser desenvolvido simultaneamente em cinco eixos estruturantes: (1) Números e Operações; (2) Pensamento Algébrico; (3) Espaço e Forma; (4) Grandezas e Medidas; e (5) Tratamento da Informação. Assim como nas abordagens estrangeiras, o pensamento algébrico no Brasil ainda está fortemente associado com a ideia de sequências e combinações de objetos.

\section{Aritmética Generalizada}

Destacamos que as duas vertentes do pensamento algébrico propostas por Blanton e Kaput (2005) ampliam consideravelmente a noção de pensamento algébrico. Vamos nos concentrar, em um primeiro momento, na Aritmética Generalizada. Blanton e Kaput (2005) abordam 
vários tipos de situações que demandam este tipo de pensamento, destacando principalmente as propriedades e relações entre números inteiros, as propriedades entre as operações, o uso da igualdade como uma relação entre quantidades, a estrutura dos números e a resolução de expressões numéricas com número desconhecido em falta.

Um aspecto que acabou se destacando muito na vertente da Aritmética Generalizada, foi o que alguns autores (CARPENTER et al., 2005; IRWIN e BRITT, 2006; FUJII e STEPHENS, 2008; STEPHENS e WANG, 2008) chamam de pensamento relacional, que tem como foco o estudo das relações entre os números, e também entre as operações matemáticas. Alguns autores (STEPHENS e WANG, 2008; IRWIN e BRITT, 2006) afirmam que o pensamento relacional se caracteriza pela compensação e pela equivalência na resolução de problemas matemáticos.

Fujii e Stephens (2008) afirmam que o pensamento relacional se caracteriza pela exploração de padrões de variação sem necessariamente apresentar representações envolvendo expressões algébricas. Os autores ressaltam que este tipo de pensamento pode ser visto como uma protoÁlgebra, ou seja, um pensamento algébrico sem representação sofisticada.

Carpenter et al. (2005), ao abordar o pensamento relacional, propõem o uso de problemas do tipo $\mathrm{a}+\mathrm{b}=+\mathrm{c}$ para auxiliar no processo de desenvolvimento deste tipo de pensamento. Segundo o autor, situações desta natureza oportunizam ao estudante pensar na igualdade não apenas como um resultado, mas como uma relação entre quantidades.

Stephens e Wang (2008), em um estudo que analisou o pensamento relacional de estudantes do $6^{\circ}$ e $7^{\circ}$ anos escolares em Portugal, utilizaram questões abordando o pensamento relacional. Além do tipo de questões utilizadas por Carpenter et al. (2005), eles também utilizaram problemas envolvendo a multiplicação e a divisão. As conclusões do trabalho de Stephens e Wang (2008) são semelhantes às de Carpenter et al. (2005), ou seja, problemas aritméticos envolvendo o pensamento relacional promovem uma visão diferenciada da operação de igualdade, que leva o estudante a considerá-la não apenas como indicativo de resultado, mas também como uma relação entre as quantidades.

\section{Pensamento Funcional}

Já abordamos algumas das principais discussões a respeito da Aritmética Generalizada, vamos agora nos concentrar no que Blanton e Kaput (2005) denominam pensamento funcional. O pensamento funcional está mais associado à ideia de descrição da variação de quantidades, que é a mesma ideia do conceito de função em matemática. Blanton e Kaput (2005) 
apresentam vários exemplos de problemas que podem auxiliar no desenvolvimento deste tipo de pensamento, destacando principalmente a simbolização das quantidades, as operações com expressões simbólicas, a representação gráfica de dados, a descoberta de relações funcionais e a previsão de resultados desconhecidos utilizando dados conhecidos.

Embora o conceito de função seja tratado mais formalmente apenas no final do Ensino Fundamental e/ou início do Ensino Médio, existem situações-problema e tarefas que podem ser realizadas desde os anos iniciais do Ensino Fundamental com o objetivo de desenvolver o pensamento funcional nos estudantes. A seguir são apresentadas as pesquisas de Ponte e Velez (2011), e também de Silva e Savioli (2012), que ilustram esta possibilidade.

Ponte e Velez (2011) analisaram as representações de dois estudantes de 7 anos em 3 tarefas envolvendo sequências e combinações de objetos. Os autores constataram que as crianças conseguiram utilizar boas representações para as sequências, conseguiram prever termos próximos do início, e em alguns casos, até mesmo termos mais afastados, seguindo o raciocínio esperado de acordo com a proposta da tarefa. No entanto, destaca-se que algumas representações podem indicar erroneamente um bom desenvolvimento do processo de conceitualização, citando o caso de um dos estudantes, que elaborou uma representação para as combinações sem, no entanto, produzir explicações que evidenciassem o entendimento do raciocínio utilizado.

Silva e Savioli (2012) utilizaram o método de Análise do Conteúdo para compreender o pensamento algébrico nas resoluções de tarefas realizadas com 35 alunos do $5^{\circ}$ ano do Ensino Fundamental. As autoras concluíram que embora os estudantes não apresentem uma linguagem simbólica para formalizar o pensamento algébrico, eles possuem mecanismos de representação para visualizar a comparação de quantidades, abstraindo algumas relações matemáticas do contexto apresentado. Em uma das tarefas aplicadas na pesquisa, não havia uma contextualização, apenas instruções que mencionavam um procedimento numérico. Nesta última tarefa, ainda que não houvesse um contexto para que as crianças o representassem, elas identificaram padrões e algumas conseguiram compreender e prever facilmente os valores que deveriam preencher nas lacunas em branco, conforme a demanda da tarefa.

Com estas duas pesquisas, finalizamos a apresentação do estado da arte sobre o pensamento funcional, e assim, fechamos a descrição das duas abordagens do pensamento algébrico indicadas por Blanton e Kaput (2005). Ressalta-se que embora novas abordagens do pensamento algébrico tenham surgido nos últimos anos, há predominância da abordagem que o considera sendo constituído estritamente por problemas que envolvem sequências e combinações com termos desconhecidos. 


\section{Considerações Finais}

A partir do estado da arte apresentado nas seções precedentes, pode-se dizer que embora tenham surgido muitos trabalhos abordando a temática do pensamento algébrico nos últimos anos, prevalece a ramificação estabelecida por Blanton e Kaput (2008), que divide o pensamento algébrico em duas vertentes: a Aritmética generalizada e o pensamento funcional. Além desta divisão em vertentes, muitos trabalhos também apresentam a ideia de que problemas que envolvem sequências e combinações potencializam a habilidade de pensar algebricamente. Com relação a referências teóricas sobre o pensamento algébrico, foi constatado que as vertentes apresentadas por Blanton e Kaput (2008) continuam exercendo influência em muitos trabalhos recentes sobre o pensamento algébrico (BRIZUELA, MARTINEZ e CAYTON-HODGES, 2013; HEUVEL-PANHUIZEN, KOLOVOU e ROBITZSCH, 2013; CASTRO G, 2014; HUNTER, 2014; McNEIL, FYFE e DUNWIDDIE, 2014; RADFORD, 2014; SILVA e SAVIOLI, 2014; BYRD, McNEIL, CHESNEY e MATTHEWS, 2015).

Com base no estado da arte das pesquisas que abordam o pensamento algébrico, propomos a seguinte metodologia de análise de dados, quando estes se referem à estratégias apresentadas por estudantes para resolver problemas que demandam uma pensamento algébrico. Denominamos tal metodologia de Análise do Potencial Algébrico de Problemas, a qual consiste em cinco etapas, descritas a seguir:

$\left.1^{\circ}\right)$ Classificação do problema, seguindo algum referencial teórico específico;

$2^{\circ}$ ) Análise dos erros cometidos na resolução;

$3^{\circ}$ ) Descrição das estratégias eficazes;

$4^{\circ}$ ) Tentativa de se identificar um pensamento algébrico, guiando-se pelo que caracteriza esta forma de pensamento (BLANTON e KAPUT, 2005; CARPENTER et al., 2005; IRWIN e BRITT, 2006; CANAVARRO, 2007; FUJII e STEPHENS, 2008; STEPHENS e WANG, 2008); e

$5^{\circ}$ ) Sistematização ou classificação das estratégias bem-sucedidas, levando em consideração as características algébricas observadas, o referencial teórico adotado e a natureza dos conceitos envolvidos no problema.

Para concluir, podemos dizer que ainda existem muitas questões em aberto sobre o desenvolvimento do pensamento algébrico, tanto no âmbito da pesquisa, quanto no contexto didático escolar, e que ainda por anos, este tema possui um amplo leque de possibilidades de estudos, constituindo também um desafio para a formação e aperfeiçoamento docente. 


\section{Referências}

BAUMGART, J. K. Álgebra. Editora Atual, São Paulo, 1992. Coleção Tópicos em sala de aula para uso em sala de aula - Volume 4. Tradução de Higino H. Domingues. 112p.

BLANTON, Maria L.; KAPUT, James J. Characterizing a classroom practice that promotes algebraic reasoning. Journal for Research in Mathematics Education, v.36, n.5, p.412-446, 2005.

BRASIL. Elementos Conceituais e Metodológicos para os Direitos de Aprendizagem e Desenvolvimento do Ciclo de Alfabetização (1 ${ }^{\circ}, 2^{\circ}$ e $3^{\circ}$ anos) do Ensino Fundamental. Ministério da Educação, Secretária de Educação Básica, Brasília, 2012.

BRIZUELA, B.M.; MARTINEZ, M.V.; CAYTON-HODGES, G.A. The Impact of Early Algebra: Results from a Longitudinal Intervention. Journal of Research in Mathematics Education, v.2, n.2, p.209-241, 2013.

BYRD, Carolyne E.; McNEIL, Nicole M.; CHESNEY, Dana L.; MATTHEWS, Percival G. A specific misconception of the equal sign acts as a barrier to children's learning of early algebra. Learning and Individuals Differences, v.38, p.61-67, 2015.

CANAVARRO, Ana Paula. O Pensamento Algébrico na Aprendizagem Matemática nos Primeiros Anos. Quadrante, v.16, n.2, p.81-118, 2007.

CAPES. Sistema WebQualis. Disponível $<$ http://qualis.capes.gov.br/webqualis/publico/pesquisaPublicaClassificacao.seam>. Acesso em: 02 Mai. 2015.

CARRAHER, David W.; MARTINEZ, Mara V.; SCHLIEMANN. Early algebra and mathematical generalization. ZDM - The International Journal on Mathematics Education, v.40, p.3-22, 2008.

CARPENTER, T. P.; LEVI, L.; FRANKE, M. L. ZERINGUE, J. K. Algebra in the elementary school: developing relational thinking. ZDM - The International Journal on Mathematics Education, v.37, n.1, p.53-59, 2005.

CASTRO G, Walter F. Razonamiento algebraico elemental: propuestas para el aula. Rerevista Científica, n.20, 2014.

FALCÃO, Jorge Tarcísio da Rocha. Alfabetização Algébrica nas Séries Iniciais. Como Começar?. Boletim GEPEM, n.42, Fev./Jul., p.27-36, 2003.

FUJII, T.Probing Students' Understanding of Variables trough Cognitive Conflict Problems: Is the Concept of a Variable So Difficult for Students to Understand? In: PATEMAN, A.; DOUGHeRTy, B. J. ZILLIOX, J. T. (Eds). Proceedings of the 27th Conference of the International Group for the Psychology of Mathematics Education, (pp.49-65). PME, Honolulu, 2003.

; STEPHENS, M. Using number sentences to introduce the idea of variable. In: GREENES, C.; RUBENSTEIN, R. (Eds). Algebra and algebraic thinking in school: 
Seventieth Yearbook, (pp.127-149). National Council of Teachers of Mathematics. VA, Reston, 2008.

GOMES, Maria da Conceição Vieira. Álgebra, Geometria e Aritmética de Mãos Dadas no Ensino Fundamental. Boletim GEPEM, n.42, Fev./Jul., p.47-59, 2003.

GREENES, C.; RUBENSTEIN, R. (Eds). Algebra and algebraic thinking in school: Seventieth Yearbook, (pp.127-149). National Council of Teachers of Mathematics. VA, Reston, 2008.

HEUVEL-PANHUIZEN, Marja van den; KOLOVOU, Angeliki; ROBITZSCH, Alexander. Primary school students' strategies in early algebra problem solving supported by an online game. Education Studies in Mathematics, v.84, p.281-307, 2013.

HUNTER, Jodie. Development learning environments which support early algebraic reasoning: a case from a New Zealand primary classroom. Mathematical Education Research Journal, v.26, p.659-682, 2014.

IFRAH, Georges. História Universal dos Algarismos. Rio de Janeiro: Editora Nova Fronteira, 1998. 1050p.

IRWIN, K. C.; BRITT, M. S. The algebraic nature of students' numerical manipulation in the New Zeland Numeracy Project. Education Studies in Mathematics, v.58, n.2, p.169-188, 2005.

KAMII, Constance. A criança e o número: implicações da teoria de Piaget. Editora Papirus, Campinas, 1990.

KAPUT, James; CARRAHER, David; BLANTON, Maria (Eds.). Algebra in the Early Grades. Lawrence Erlbaum Associates, Mahwah-NJ, 2007. 552p.

KIERAN, Carolyn. Developing algebraic reasoning: The role of sequenced tasks and teacher questions from the primary to the early secondary school levels. Quadrante, v.16, n.1, p.5-26, 2007.

LINZ, R. C.; GIMENEZ, J. Perspectivas em Aritmética e Álgebra para o Século XXI. Campinas: Editora Papirus, 2006. 176p.

McNEIL, Nicole M.; FYFE, Emily R.; DUNWIDDIE, April E. Arithmetic Practice Can Be Modified to Promote Understanding of Mathematical Equivalence. Journal of Educational Psychology, 2014.

MOLINA, M. La integración de pensamiento algebraico em Educación Primária. En: CAMACHO, M.; FLORES, P.; BOLEA (Eds.), Investigación en educación matemática XI (pp. 53-69). Tenerife: Caja Canarias y Sociedad Espanõla de In-vestigación en Educación Matemática - SEIEM, 2009.

NCTM. 2000. Princípios e Normas para a Matemática Escolar. (1.ed. 2000) Tradução portuguesa dos Principles and Standards for School Mathematics. 2.ed. Lisboa: APM, 2008. 
PIAGET, Jean. 1937. A Construção do Real na Criança. Editora Zahar, Rio de Janeiro, 1979.

, Jean. 1950. Introduction à l'épistemologie génétique: la pensée mathématique. Presses Universitaire de France, Paris, 1950. v.1.

, Jean. 1977. Abstração Reflexionante. Editora Artmed, Porto Alegre, 1990.

PONTE, João Pedro da; VELEZ, Isabel. Representações em tarefas algébricas no $2^{0}$ ano de escolaridade. Boletim GEPEM, n.59, Jul./Dez., p.53-68, 2011.

RADFORD, Luis. The Progressive Development of Early Embodied Algebraic Thinking. Mathematical Education Research Journal, v.26, p.257-277, 2014.

ROMANOWSKI, Joana Paulin; ENS, Romilda Teodora. As pesquisas denominadas "estado da arte" em educação. Revista Diálogo Educacional, v.6, n.19, Set./Dez., p.37-50, 2006.

SILVA, Daniele Peres; SAVIOLI, Angela Marta Pereira das Dores. Caracterizações do pensamento algébrico em tarefas realizadas por estudantes do Ensino Fundamental I. Revista Eletrônica de Educação, v.6, n.1, p.206-222, 2012.

SILVA, Daniele Peres; SAVIOLI, Angela Marta Pereira das Dores. Manifestações do Pensamento Algébrico em Resoluções de Tarefas por Estudantes do Ensino Fundamental I. Revista Paranaense de Educação Matemática, v.3, n.5, p.139-156, 2014.

SOARES, June; BLANTON, Maria L; KAPUT, James J. Thinking Algebraically across the Elementary School Curriculum. Teaching Children Mathematics, NCTM, p.228-235, 2006.

STEPHENS, M.; WANG, X. Investigating some junctures in relational thinking: a study of year 6 and 7 students from Australia and China. Journal of Mathematics Education, v.1, n.1, p.28-39, 2008.

VERGNAUD, Gérard. 1985. A criança, a matemática e a realidade: problemas do ensino da matemática na escola elementar. Tradução de Maria Lucia Faria Moro. 3ed. Editora da UFPR, Curitiba, 2009.

, Gérard. La théorie des champs conceptuels. Recherches em Didactique des Mathématiques, v.10, n.2-3, p.133-170, 1990.

Gérard. The nature of mathematical concepts. In NUNES, T. \& BRYNT, P. (Eds.) Learning and teaching mathematics, an international perspective. Psychology Press Ltd, Hove (East Sussex), 1997.

VERSCHAFFEL, L.; GREER, B.; De CORTE, E. Whole number concepts and operations . In LESTER, F. K. (Ed.) Second Handbook of Research on Mathematics Teaching and Learning, p.557-628, Charlotte, NC: NCTM \& Information Age Publishing, 2007. 